Harold A. McAlister and William I. Harkopf (eds.)

\title{
Spectroscopic and Photometric Studies of an X-ray-Selected Sample of Chromospherically Active Binary Stars ${ }^{1}$
}

\author{
ROBERT P. STEFANIK \\ Harvard-Smithsonian Center for Astrophysics, 60 Garden St., \\ Cambridge, MA 02138, USA \\ LAURENCE A. MARSCHALL ${ }^{2}$ \\ Dept. of Physics, Gettysburg College, Gettysburg, PA 17325, USA \\ HAROLD L. NATIONS ${ }^{2}$ \\ The College of Charleston, Charleston, SC 24924, USA
}

\section{INTRODUCTION}

Data from the Einstein X-Ray satellite continue to provide useful information for studies of $\mathbf{x}$-ray emitting objects. The Einstein Medium Sensitivity Survey (EMSS), a tabulation of serendipitously discovered point sources from the Einstein database, included a number of objects which were identified, on the basis of spot sampling of optical spectra, as likely binary star systems (Fleming 1988, Silva et al. 1987; Takalo \& Nousek 1988). Because the sample is limited primarily by X-ray flux, the physical characteristics of these stars are of considerable interest for understanding the origins of stellar activity among cool stars.

\section{OBSERVATIONS}

For four years we have been conducting a spectroscopic and photometric study of about four dozen of these stars to determine the orbital and physical characteristics of the individual members of the sample, to see if they represent a single population, and to discover objects of interest for the study of activity in late-type binary systems.

Our observations to date include the following:

1. Radial velocity observations have been obtained using the echelle spectrographs at Oak Ridge Observatory, and Fred L. Whipple Observatory. A few additional observations have been made using the MMT. Spectra are reduced at the Center for Astrophysics using standard CfA correlation reduction techniques, and orbital solutions are obtained using analysis programs written by Dr. Tzevi Mazeh. The stars we have best results on to

\footnotetext{
'Some of the obeervations reported were obtained at the Multiple Mirror Telescope Observatory, a joint facility of the Smitheonian Institution and the University of Arirona.

${ }^{2}$ Visiting Astronomer, Kitt Peak National Observatory, National Optical Astronomy Observatories, operated by the Association of Universities for Research in Astronomy, Inc., under contract with the National Science Foundation.
} 
date are those for which we have obtained good correlations using CfA templates, i.e. those with small to moderate Vsini (roughly $<50 \mathrm{~km} / \mathrm{s}$ ). New reduction procedures using synthetic spectra matched to the rotationally broadened stellar spectra should make it possible to obtain reliable orbits for other stars for which we have numerous spectra, but whose solutions are not yet secure.

2. Photometric observations have been obtained using photoelectric photometers at Grundy Observatory, Gettysburg College, and the National Undergraduate Research Observatory, and CCD cameras at the Kitt Peak National Observatory. These observations have revealed several eclipsing binaries, and are also being used to study rotational starspot modulation of the light from the binary components. We have reported so far on two eclipsing binaries, 1E1247-0548, a detached system, and 1E2038-0046, a near contact binary. (Marschall et al. 1989; Marschall et al. 1991).

3. Additional high-resolution spectra have been obtained using the coudé feed at KPNO and the cassegrain spectrograph on the 72-inch reflector at Lowell Observatory to study the extent and variability of activity among these stars, and to detect secondaries that are unresolved in CfA spectra.

The status of our observations to date is summarized in Table 1. The first 5 columns list vital statistics on the stars. Values of Vsin $i$, when specified, are taken from Fleming's thesis (Fleming 1988). Column 6 lists the number of spectroscopic observations taken at CfA. Comments in column 7 include notes on whether the object is a confirmed or suspected single line (SB1) or double line (SB2) binary, whether the orbit is circular $(-\mathrm{O})$, and what period has been determined, if any.

\section{DISCUSSION}

From our spectroscopic and photometric observations of several dozen X-ray selected stars from the Einstein Medium Sensitivity Survey we can report the following:

- We have derived new spectroscopic orbits for 12 stars.

- Most of the binary candidate stars selected from the EMSS by Fleming (1988), Silva et al. (1987), and Takalo \& Nousek (1988), are in fact binary stars and will in time yield orbital solutions.

- Most orbits we have observed so far have periods between 1 and 10 days.

- The eccentricities of most of the orbits are very low or zero. The shortest period non-circular orbit is 7 days $(1 \mathrm{E} 08240, \mathrm{P}=6.95 \mathrm{~d}, \mathrm{e}=0.4)$, but circular orbits with periods of 9.5 days (1E1937) and 11 days (1E15208) are also found.

- We have discovered two eclipsing binaries so far: 1E 1247-0548 (HD111487) and 1E2038-0046 (HD197010). The former appears to be a detached system, possibly an RS CVn binary, and the latter appears to be a nearcontact binary system. We are looking for photometric variability in the other systems, both to detect eclipsing systems and to discover rotational modulation of the component stars, a phenomenon evident on 1E2038-0046 (Marschall et al. 1991). 
TABLE 1. EMSS X-ray Binary Candidates Observed at CfA (as of 4/1/1992)

\begin{tabular}{|c|c|c|c|c|c|c|}
\hline Name & R.A., Dec. (1950) & Mag & $V \sin i$ & SAO & $\mathbf{N}$ & Comments \\
\hline $\begin{array}{l}1 \mathrm{E} 00029 \\
1 \mathrm{E} 00099\end{array}$ & $\begin{array}{l}00: 02: 52+16: 02: 55 \\
00: 09: 54+14: 17: 17\end{array}$ & $\begin{array}{l}8.8 \\
8.5\end{array}$ & $\begin{array}{l}12 \\
22\end{array}$ & $\begin{array}{l}091699 \\
091772\end{array}$ & $\begin{array}{l}39 \\
36\end{array}$ & $\begin{array}{l}\text { SB2? SB1-O, } \mathrm{P}=295.66 \\
\text { SB1-O, triple?, } \mathrm{P}= \\
1.844 \text { (Latham 1988) }\end{array}$ \\
\hline $1 \mathrm{E} 00116$ & $00: 11: 38+08: 40: 58$ & 11.5 & 22 & - & 34 & SB2, SB3?, $P=25 ?$ \\
\hline $1 \mathrm{E} 01053$ & $01: 05: 16+31: 44: 54$ & 6.3 & 68 & 054445 & 11 & \\
\hline $1 E 01344$ & $01: 34: 25+20: 27: 11$ & 8.7 & 11 & 074827 & 49 & $S B 2-O, P=25.34$ \\
\hline $1 E 02342$ & $02: 34: 12-03: 21: 49$ & 8.1 & $<10$ & 130011 & 45 & $S B 1-O, P=14.83$ \\
\hline $1 \mathrm{E} 02417$ & $02: 41: 40+10: 45: 05$ & 11.1 & 28 & - & 15 & SB? \\
\hline $1 E 02449$ & $02: 44: 51-00: 24: 57$ & 9.6 & 17 & 130113 & 40 & $\mathrm{SB} 1-\mathrm{O}, \mathrm{P}=2.63$ \\
\hline $1 E 03158$ & $03: 15: 48-19: 55: 15$ & 10.8 & 23 & - & 10 & Constant? \\
\hline $1 E 03267$ & $03: 26: 41-20: 08: 38$ & 8.9 & 12 & - & 31 & SB1-O, $P=3.18$ \\
\hline $1 \mathrm{E} 03482$ & $03: 48: 13-14: 04: 20$ & 10.7 & $<10$ & - & 26 & $\mathrm{SB} 2, \mathrm{SB} 1-\mathrm{O}, \mathrm{P}=9.3$ \\
\hline $1 \mathrm{E} 04294$ & $04: 29: 22+17: 55: 11$ & 12.1 & $<10$ & - & 16 & $\begin{array}{l}\mathrm{SB2}, \mathrm{P}=3.89 \\
\text { (Reipurth et al. } 1990 \text { ) }\end{array}$ \\
\hline $1 \mathrm{E} 04386$ & $04: 38: 33+02: 13: 05$ & 10.7 & - & - & 29 & SB? \\
\hline $1 \mathrm{E} 05050$ & $05: 05: 01-05: 27: 55$ & 10.2 & 14 & - & 14 & $\begin{array}{l}\text { SB1-O, } P=9.80 \text {, } \\
\text { (Fleming 1988) }\end{array}$ \\
\hline $\begin{array}{l}1 \mathrm{E} 0657 \mathrm{~N} \\
1 \mathrm{E} 0657 \mathrm{~S}\end{array}$ & $06: 57: 29+75: 18: 26$ & 8.3 & $<10$ & 006052 & $\begin{array}{c}11 \\
9\end{array}$ & $\begin{array}{l}\text { Constant velocity } \\
\text { SB1 }\end{array}$ \\
\hline $1 \mathrm{E} 07303$ & $07: 30: 20+65: 46: 59$ & 8.4 & 17 & 014241 & 21 & SB1? \\
\hline $1 E 08240$ & $08: 24: 01+29: 44: 39$ & 8.6 & $<10$ & 080190 & 48 & SB1-O, $P=6.95$ \\
\hline $1 E 08427$ & $08: 42: 39+19: 00: 01$ & 6.8 & $<10$ & 098098 & 33 & Constant velocity \\
\hline $1 \mathrm{E} 09243$ & $09: 24: 20+39: 42: 45$ & 9.7 & $<10$ & - & 39 & SB2-O, $P=8.49$ \\
\hline $1 \mathrm{E} 09568$ & $09: 56: 50-22: 25: 14$ & 9.2 & 15 & 178272 & 31 & SB2-O, $P=1.84$ \\
\hline $1 E 10226$ & $10: 22: 36+11: 21: 26$ & 10.6 & 58 & - & 27 & SB \\
\hline $1 \mathrm{E} 10495$ & $10: 49: 28-08: 49: 21$ & 11.2 & - & - & 15 & SB \\
\hline 1E10502 & $10: 50: 09-09: 25: 28$ & 12.5 & 11 & - & 4 & SB \\
\hline $1 E 11279$ & $11: 27: 53-15: 02: 47$ & 9.4 & 21 & 156720 & 41 & $\mathrm{SB} 2, \mathrm{P}=2.3 ?$ \\
\hline $1 \mathrm{E} 12086$ & $12: 08: 37+39: 24: 47$ & 8.0 & var & 062883 & 12 & Velocity variable \\
\hline $1 \mathrm{E} 12225$ & $12: 22: 31+25: 49: 40$ & 8.1 & - & 082295 & 62 & $\begin{array}{l}\text { SB2-O, P=0.96 } \\
\text { (Kraft 1965) }\end{array}$ \\
\hline 1E12470 & $12: 47: 03-05: 48: 22$ & 9.0 & - & 138983 & 42 & SB1; sec res? eclipsing \\
\hline $1 E 14369$ & $14: 36: 52-26: 28: 48$ & 9.2 & - & 182743 & 16 & SB1? \\
\hline 1E14404 & $14: 40: 24+52: 13: 25$ & 7.5 & 70 & 029248 & 7 & Velocity variable? \\
\hline $1 \mathrm{E} 15208$ & $15: 20: 47-06: 25: 48$ & 7.3 & 35 & 140499 & 20 & $\begin{array}{l}\text { SB1-O, P=11.13 } \\
\text { (Fekel et al. } 1985 \text { ) }\end{array}$ \\
\hline $1 E 15306$ & $15: 30: 37+13: 42: 55$ & 12.4 & 21 & - & 7 & Velocity variable \\
\hline 1E15330 & $15: 33: 00+09: 19: 08$ & 11.7 & 30 & - & 24 & $\mathrm{SB}, \mathrm{P}=2.5 ?$ \\
\hline 1E15488 & $15: 48: 45+11: 25: 16$ & 12.8 & 30 & - & 9 & Velocity Variable \\
\hline 1E16150 & $16: 14: 58+31: 14: 15$ & 12.6 & 95 & - & 1 & \\
\hline 1E16540 & $16: 53: 59+35: 15: 38$ & 12.3 & 95 & - & $i$ & \\
\hline $1 \mathrm{E} 17373$ & $17: 37: 16+68: 47: 10$ & 4.8 & $<10$ & 017576 & 33 & $\begin{array}{l}\text { SB2-O, } P=5.3 \\
\text { (Abt \& Levy 1976) }\end{array}$ \\
\hline 1E17510 & $17: 51: 03+70: 46: 17$ & 9.6 & 30 & - & 22 & SB1 \\
\hline $1 \mathrm{E} 1806 \mathrm{~N}$ & $18: 06: 03+69: 44: 51$ & 10.5 & 100 & - & 1 & \\
\hline IE1806S & & & & & 1 & \\
\hline 1E18481 & $18: 48: 10+33: 05: 30$ & 10.0 & 24 & - & 62 & SB2; $P<2 d$ ?; triple? \\
\hline $1 \mathrm{E} 19371$ & $19: 37: 04+30: 27: 58$ & 10.0 & 35 & - & 40 & $\mathrm{SB} 1 ; \mathrm{P}=9.52 \mathrm{~d}$ \\
\hline $1 E 20383$ & $20: 38: 20-00: 46: 26$ & 9.4 & 100 & 144699 & 9 & Near-contact, eclipsing \\
\hline $1 \mathrm{E} 21134$ & $21: 13: 24+05: 17: 09$ & 12.8 & $<10$ & - & 6 & Velocity var, Low ampl \\
\hline $1 E 21167$ & $21: 16: 40-10: 42: 17$ & 12.1 & 60 & - & 5 & Velocity variable \\
\hline 1 E21197 & $21: 19: 44+16: 55: 32$ & 11.7 & 100 & - & 2 & \\
\hline $1 E 21483$ & $21: 48: 16+14: 20: 43$ & 14.4 & $<10$ & - & 1 & \\
\hline $1 \mathrm{E} 2346 \mathrm{~A}$ & $23: 46: 53+18: 42: 27$ & 12.1 & 21 & - & 5 & Velocity variable \\
\hline 1E2346B & & & & & 2 & \\
\hline $1 \mathrm{E} 23498$ & 23:49:51-01:12:56 & 10.7 & 50 & - & 29 & SB1? \\
\hline
\end{tabular}


We are continuing observations of the stars in this sample to derive further orbits and to define the photometric and spectroscopic characteristics of the individual binaries.

\section{ACKNOWLEDGMENTS}

Many people have contributed to this work over the past several years. We would like to acknowledge echelle observers Jim Peters, Bob Davis, Joe Zajac, Joe Costello, and Ed Horine. Our students Chris Gauthier, Tracy Huard, Brian Taylor, and Kevin Witman provided additional photometric observations; Mike Divittorio provided technical support; David Latham and Tom Fleming supplied encouagement. Observations were made at Kitt Peak National Observatory, Fred L. Whipple Observatory, Oak Ridge Observatory, the National Undergraduate Research Observatory (which operates an $0.8 \mathrm{~m}$ telescope owned by the Lowell Observatory), Grundy Observatory (Franklin and Marshall College), and the Gettysburg College Observatory. This research was supported in part by grants from Gettysburg College.

\section{REFERENCES}

Abt, H. \& Levy, S.G. 1976, ApJS, 30, 273

Fekel, F., Hall, D.S., Africano, J.L, Gillies, K., Quigley, R., \& Fried, R. 1985, AJ, 90, 2581

Fleming, T. 1988 , Ph.D. thesis, University of Arizona

Kraft, R. 1965, ApJ, 142, 695

Latham, D.W., Maseh, T., Carney, B.W., McCrosky, R.E., Stefanik, R.P., \& Davis, R.J. 1988, $A J, 96,567$

Marschall, L.A., Nations, H, \& Witman, K. 1989, BAAS, 20, 994

Marschall, L.A., Gauthier, C.P., Nations, H.L., Taylor, B.W., \& Huard, T.L. 1991, Inf. Bull. Var. Stars, 3633

Reipurth, B., Lindgren, H., Nordstrom, B., \& Mayor, M. 1990, $A \& A, 235,197$

Silva, D.R., Gioia, I.M., Maccacaro, T., Mereghetti, S., \& Stocke, J.T. 1987, AJ, 93, 869

Stocke, J.T. $1987, A J, 93,869$

Takalo, L.O.\& Nousek, J.A. 1988, ApJ, 326, 779 